\title{
OPA1 Deficiency Associated with Increased Autophagy in Retinal Ganglion Cells in a Murine Model of Dominant Optic Atrophy
}

\author{
Kathryn E. White, ${ }^{1,2}$ Vanessa J. Davies, ${ }^{2,3}$ Vanessa E. Hogan, ${ }^{1}$ Malgorzata J. Piechota, ${ }^{3}$ \\ Philip P. Nichols, ${ }^{1}$ Douglas M. Turnbull, ${ }^{1}$ and Marcela Votruba ${ }^{3}$
}

Purpose. To examine retinal ganglion cell (RGC) and axonal abnormalities in an ENU-induced mutant mouse carrying a protein-truncating nonsense mutation in OPA1. Mutations in the OPA1 gene cause autosomal dominant optic atrophy (ADOA) in which loss of RGCs followed by myelin degeneration in the optic nerve leads to progressive decrease in visual acuity.

Methods. Ultrastructure of the optic nerve was examined in heterozygous mutants and wild-type littermate controls at 6, 9, and 24 months using electron microscopy. The RGC layer was examined at 6 and 24 months.

Results. There was an increase in the number of autophagosomes in the RGC layer in heterozygous mutants compared with wild type at 24 months. Signs of optic nerve degeneration were seen as early as 9 months in $O p a 1^{+/-}$mice, with more severe degeneration by 24 months. By 24 months, degeneration of axons was also seen in control mice. Numbers of opaque mitochondria in the $O p a 1^{+/-}$mice increased at 6 and 24 months, possibly representing an increase in the density of cristae to fulfill the energy requirements of the axon. In addition, mitochondria with vesiculation of the inner membranes, similar to the mutant mitochondria described in a mouse model of Charcot-Marie-Tooth type 2A, were observed.

Conclusions. Mutations in OPA1 cause pathologic changes to optic nerve axons that are similar to, but occur earlier than, age-related degeneration. Increased autophagy is likely to result from an increase in abnormal mitochondria and could be one mechanism contributing to RGC loss and subsequent optic atrophy seen in ADOA. (Invest Ophthalmol Vis Sci. 2009;50: 2567-2571) DOI:10.1167/iovs.08-2913

From the ${ }^{1}$ Mitochondrial Research Group, Newcastle University, Newcastle upon Tyne, United Kingdom; and the ${ }^{3}$ Visual Neuroscience and Molecular Biology Group, School of Optometry and Vision Sciences, Cardiff University, Cardiff, United Kingdom.

${ }^{2}$ These authors contributed equally to the work presented here and should therefore be regarded as equivalent authors.

Supported in part by Medical Research Council Grant G108/523.

Submitted for publication September 19, 2008; revised November 7, 2008, and January 3, 2009; accepted April 16, 2009.

Disclosure: K.E. White, None; V.J. Davies, None; V.E. Hogan, None; M.J. Piechota, None; P.P. Nichols, None; D.M. Turnbull, None; M. Votruba, None

The publication costs of this article were defrayed in part by page charge payment. This article must therefore be marked "advertisement" in accordance with 18 U.S.C. $\$ 1734$ solely to indicate this fact.

Corresponding author: Marcela Votruba, Visual Neuroscience and Molecular Biology Group, School of Optometry and Vision Sciences, Cardiff University, Cardiff CF24 4LU, UK; votrubam@cardiff.ac.uk.
A utosomal dominant optic atrophy (ADOA) ${ }^{1}$ is the most common form of primary inherited optic neuropathy; onset generally occurs in early childhood. ${ }^{2}$ The disease is characterized by a progressive decrease in visual acuity, color vision defects, loss of sensitivity in the central visual field, and temporal optic nerve pallor. ${ }^{3}$ Pathologic examination has shown that there is a reduction in the number of retinal ganglion cells (RGCs), particularly those in the macular region, and loss of myelin in the optic nerve, suggesting that the etiology of the disease is degeneration of the RGCs with ascending optic atrophy. ${ }^{4,5}$

It has previously been shown that mutations in the OPA1 gene cause ADOA. ${ }^{6,7}$ OPA 1 is a mitochondrial protein that is localized to the inner membrane ${ }^{8}$ and, in conjunction with mitofusins Mfn1 and Mfn2, plays a major role in mitochondrial fusion. ${ }^{9,10}$ A balance between fission and fusion is essential for maintaining the integrity of the mitochondrial network. Downregulation of OPA1 results in fragmentation of the mitochondrial network, dissipation of the mitochondrial membrane potential, disorganization of the cristae, and release of cytochrome $c$, followed by caspase-dependent apoptotic nuclear events. ${ }^{11}$

We have recently generated an ENU-induced mutant mouse carrying a protein-truncating nonsense mutation in opa1, which is a valuable tool for investigating the pathophysiology of ADOA. In this model, the heterozygous mutant mice demonstrated visual function impairment and degeneration of the optic nerve. ${ }^{12}$ In addition, fibroblasts from the adult heterozygous mutant mice showed an increase in mitochondrial fission and fragmentation. The onset of optic nerve degeneration was slow, with the first abnormalities appearing at 9 months and becoming more prominent by 18 months.

The aim of this study was to determine the degree of axonal and retinal ganglion cell pathology in heterozygous Opa 1 mice to explore potential mechanisms for neurodegeneration. In view of the observation that visual loss is delayed in the mice, as in patients with ADOA, we studied mice up to 24 months of age and compared the morphologic changes with those seen in the normal aging process.

\section{Materials ANd Methods}

\section{Generation of Mice}

The genotype analysis and breeding strategy for generation of the heterozygous Opa 1 mice have been described in detail elsewhere. ${ }^{12}$ In brief, sperm from $\mathrm{C} 3 \mathrm{H}$ males were used (in vitro fertilization with C57Bl/6J females) to generate heterozygous hybrid $\mathrm{Opa1}^{+/-}$ "founders" (the B6; C3-Opa $1^{\mathrm{Q} 258 \mathrm{X}}$ mouse line). These founder mice were then crossed with $\mathrm{C} 57 \mathrm{Bl} / 6 \mathrm{JCrl}$ mice (Charles River, East Lothian, UK) to move the mutation to a $\mathrm{C} 57 \mathrm{Bl} / 6 \mathrm{JCrl}$ background. Polymerase chain reaction of tail DNA was used to distinguish wild-type and 

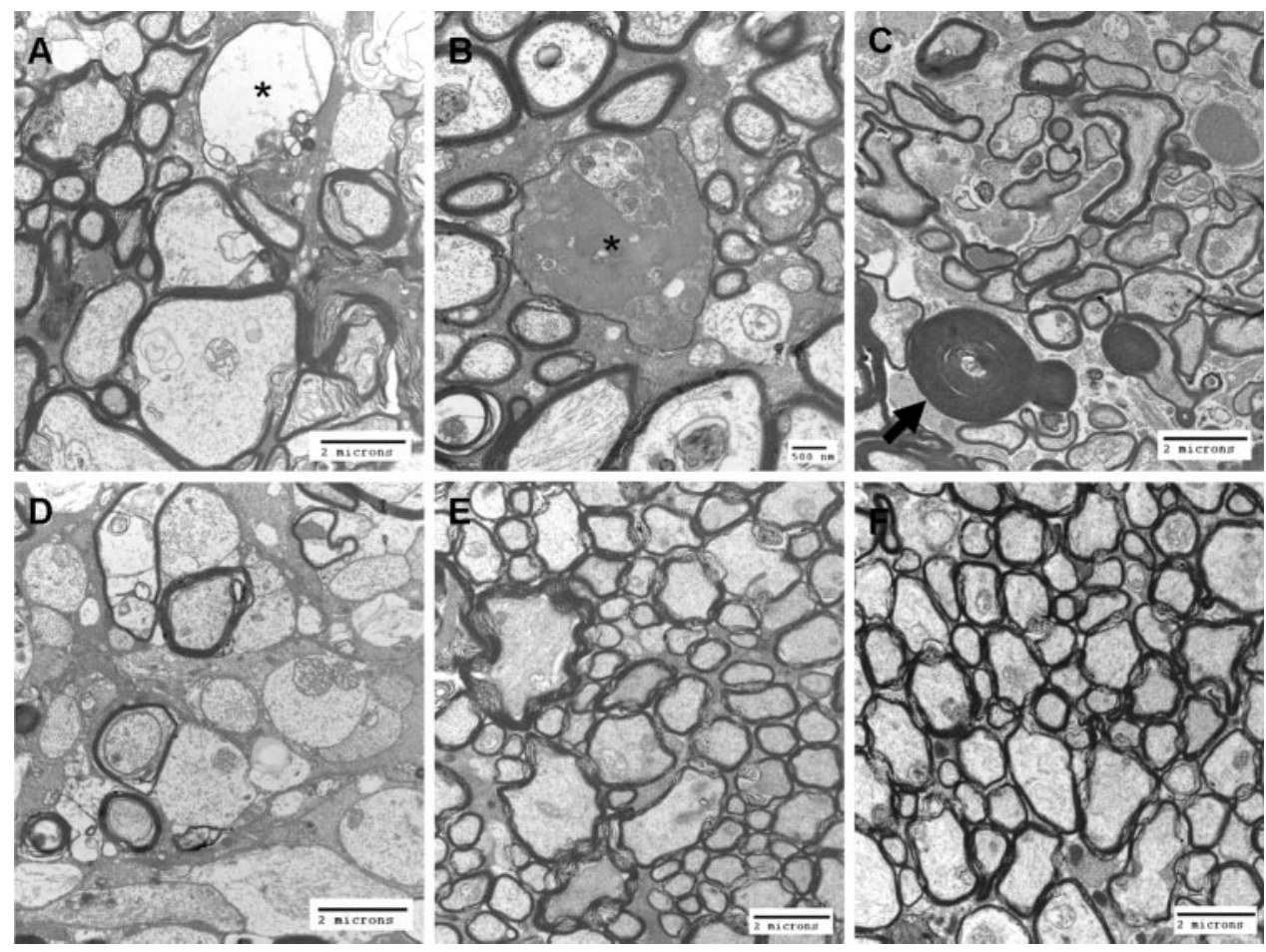

FIGURE 1. Electron micrographs of optic nerve axons from 24-month-old mice. Axon abnormalities were classified as (A) watery degeneration (asterisk), (B) dark degeneration (asterisk), (C) myelin clumping (arrow), and (D) demyelination. Areas of normal optic nerve from 24-month-old (E) and 6-month-old (F) mice.

heterozygous mice. All tested $O p a 1^{+/-}$and $O p a 1^{+/+}$mice were homozygous wild-type for the $r d l$ allele.

All experiments were approved by the animal ethics committee and were conducted in accordance with the ARVO Statement for the Use of Animals in Ophthalmic and Vision Research and the Home Office Animals in Scientific Procedures Act of 1985.

\section{Electron Microscopy}

Three $O p a 1^{+/-}$mice and three littermate wild-type controls were examined at 6 months, and five $O p a 1^{+/-}$mice and six littermate controls were examined at 24 months. The mice were killed by lethal intraperitoneal injection of phenobarbitone and were perfusion fixed with a mixture of $4 \%$ paraformaldehyde and 5\% glutaraldehyde in cacodylate buffer. The eye was dissected out, and the optic nerve and small pieces of the retina were removed. Optic nerves were also available from three $\mathrm{Opa1}^{+/-}$and three littermate controls at 9 months, though these samples were immersion rather than perfusion fixed. All samples were postfixed in osmium tetroxide, dehydrated in acetone, and embedded in epoxy resin.

\section{Optic Nerve}

Transverse ultrathin sections (approximately $90 \mathrm{~nm}$ ) were taken from the retinal end of the optic nerve, with care taken to avoid the lamina cribosa. Sections were placed on copper grids, stained with uranyl acetate and lead citrate, and examined under a transmission electron microscope (CM100; Philips, Eindhoven, Netherlands).
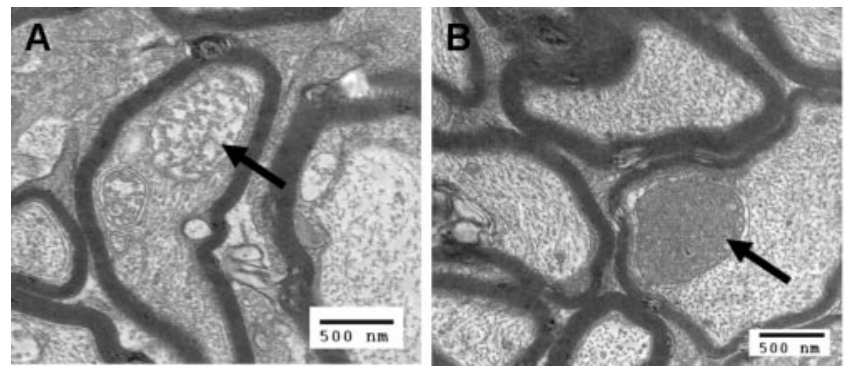

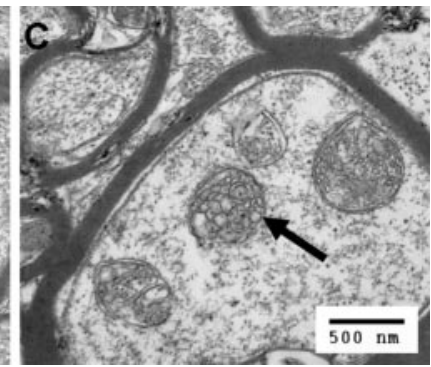

Fifteen systematically sampled random images of each optic nerve were taken at a magnification of $7900 \times$ for the analysis of axonal degeneration. An unbiased counting frame was superimposed on each image with image analysis software (Image Pro 6.2; Media Cybernetics, Bethesda, MD), and the number of axons within the frame was counted. The number of abnormal axons was also counted, with abnormalities classified as "watery" degeneration, "dark" degeneration, myelin clumping with redundant loops of myelin, or demyelination (Fig. 1). The number of abnormal axons in each classification was expressed as a percentage of the total number of axons. All axons were classified by a single masked observer $(\mathrm{VH})$. The images were also used to estimate the number of axons per area of optic nerve to determine whether there was any axonal loss.

Another 15 images were taken at a higher magnification $(\times 13,500)$ for analysis of mitochondrial morphology. Mitochondria were classified as either clear or opaque, depending on the electron density of the mitochondrial matrix (Fig. 2), and were expressed as a percentage of the total number of mitochondria analyzed. Any abnormal-appearing mitochondria were noted. All mitochondria were classified by a single masked observer (KW).

\section{Retina}

Ultrathin sections were taken through the tissue such that all the layers of the retina could be distinguished. Three blocks of retina were sectioned from each animal. On average, 45 systematically sampled random images at a magnification of $\times 4600$ were taken of the retinal
FigURE 2. Electron micrographs of optic nerve axons from 24-month-old mice. Mitochondria were classified as (A) clear or (B) opaque. (C) Occasional aberrant mitochondria with vesiculation of the inner membrane were seen in $\mathrm{Opa1}^{+/-}$mice. 
Figure 3. (A) Electron micrograph of early- and late-stage autophagy in retinal ganglion cell layer from a 24-month-old mouse. In the earlier stage, the doublemembrane bound structure (arrow) is fusing with a lysosome (arrowhead). At the later stage (asterisk), fusion with the lysosome has taken place, and the structure is undergoing proteolysis. (B) Graph showing the numbers of autophagosomes per unit area of the retinal ganglion cell layer in $O p a 1^{+/-}$and Opa1 $1^{+/+}$ mice. At 6 months, there was no difference. At 24 months, there was a significant increase in autophagosomes in the Opa1 $1^{+/-}$ mice compared with wild-type controls $(P=0.016)$. There was a significant reduction in the
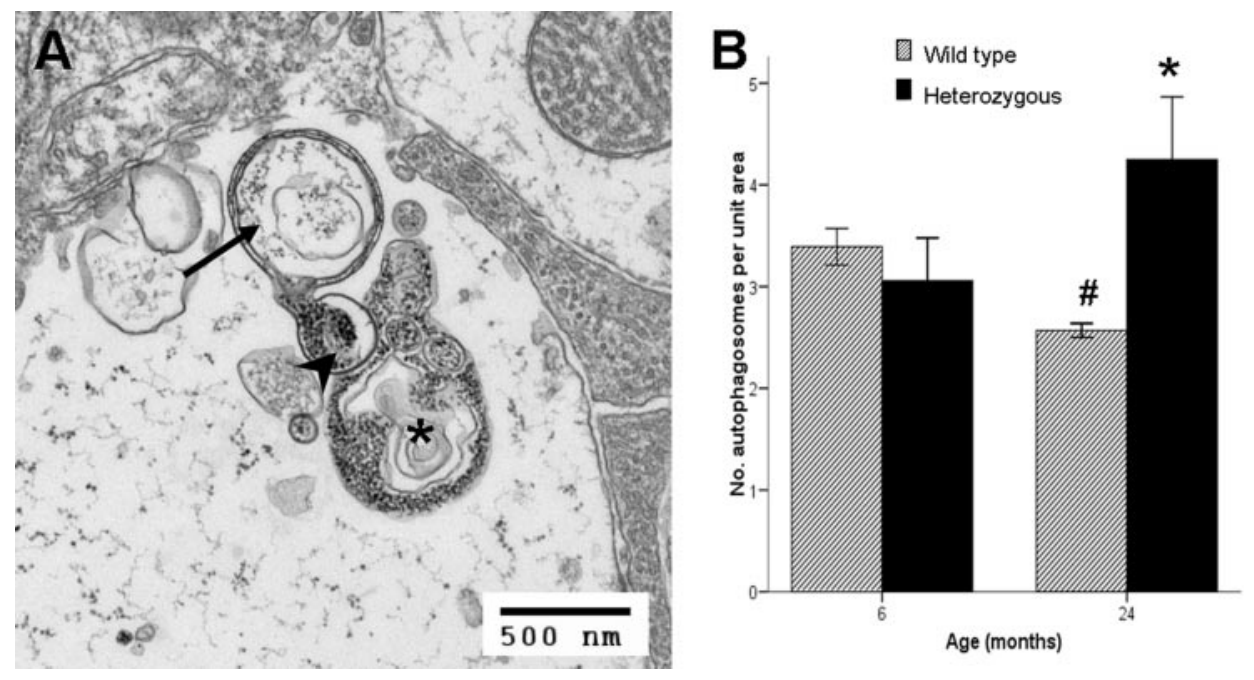
number of autophagosomes with age in the wild-type controls $(P=0.001)$. ${ }^{*}$ Significant differences between $O p a 1^{+/-}$and $O p a 1^{+/+}$ mice. \#Significant differences between 6 and 24 months.

ganglion cell layer in each mouse. Autophagosomes were observed in the ganglion cell layer and the surrounding axons. These were counted and expressed as number per unit area. An autophagosome was defined as a double membrane-bound structure that might or might not be fused with a lysosome (Fig. 3A).

\section{Statistical Analysis}

Analysis was conducted with statistical analysis software (SPSS 11.0; SPSS Inc., Chicago, IL). Comparisons between groups were performed with Student's $t$-test or ANOVA. Two-tailed $P<0.05$ was considered statistically significant.

\section{Results \\ Optic Nerve}

The quantification of structural changes seen in the optic nerve axons is illustrated in Figure 4. At 6 months, there were no differences in the structure of the axons between $O p a 1^{+/-}$
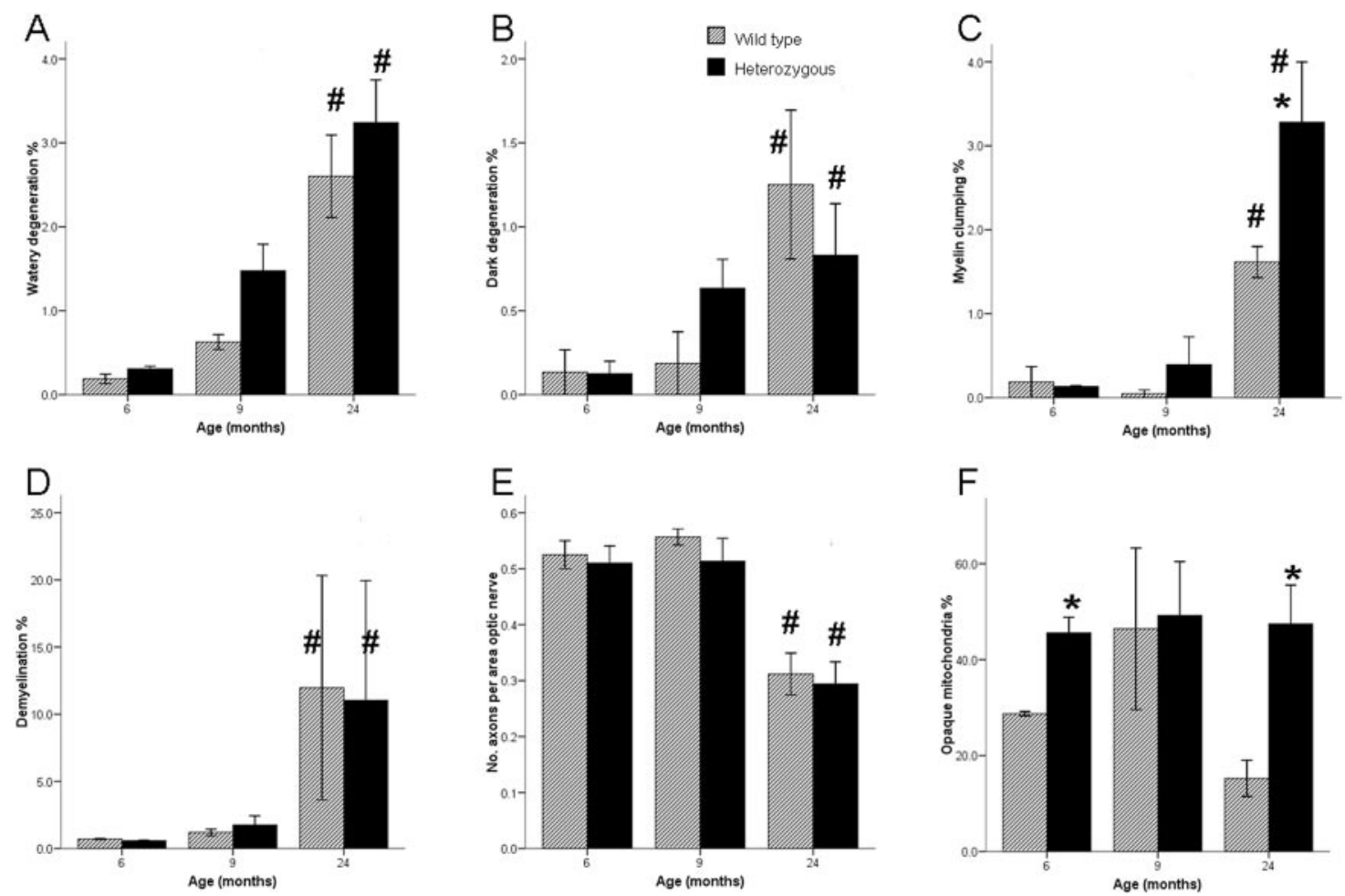

Figure 4. Graphs showing quantification of structural changes in the optic nerve axons at 6,9 and 24 months in $O p a 1^{+/-}$and $O p a 1^{+/+}$mice. There were no differences in axonal structure between $O p a 1^{+/-}$and $O p a 1^{+/+}$mice at 6 months. At 9 months, there was evidence of watery and dark degeneration in the $O p a 1^{+/-}$mice, but this did not reach statistical significance. At 24 months, there was a significant increase in the percentage of axons with disorganized myelin (clumping) in the $O p a 1^{+/-}$compared with wild-type controls $(P=0.037)$. All types of degeneration were significantly increased with age in $\mathrm{Opal}^{+/-}$and $\mathrm{Opa}^{+/+}$mice, and there was a significant reduction in the number of axons per area optic nerve, indicating axonal loss. There were increased numbers of opaque mitochondria in $O p a 1^{+/-}$compared with wild-type controls at $6(P=$ $0.027)$ and $24(P=0.004)$ months. *Significant differences between $O p a 1^{+/-}$and $O p a 1^{+/+}$. \#Age-related significant differences. 
mice and littermate controls (Figs. 4A-D). At 9 months, the Opa $1^{+/-}$mice were beginning to show evidence of watery and dark degeneration compared with littermate controls (Figs. 4A, B), though these differences did not reach statistical significance $(P=0.06, P=0.08$, respectively). At 24 months, $O p a 1^{+/-}$mice had a significantly higher percentage of axons with disorganized myelin (clumping) than did littermate controls $(3.28 \% \pm 1.60 \%$ vs. $1.62 \% \pm 0.45 \% ; P=0.037$; Fig. $4 \mathrm{C})$. There was no difference in the number of axons per area of optic nerve between $O p a 1^{+/+}$and $O p a 1^{+/-}$mice at 6,9, and 24 months (Fig. 4E).

Mitochondria with clear matrix and opaque matrix were found in $O p a 1^{+/-}$and Opa $1^{+/+}$mice. However, the percentage of opaque mitochondria was significantly increased in the Opa $1^{+/-}$mice at 6 and 24 months $(45.58 \% \pm 5.60 \%$ vs. $28.70 \%$ $\pm 0.66 \%[P=0.027]$ and $47.43 \% \pm 18.14 \%$ vs. $15.25 \% \pm 9.24 \%$ $[P=0.004]$, respectively; Fig. $4 \mathrm{~F})$. Some mitochondria in the optic nerves of 24 -month-old $O p a 1^{+/-}$mice had structural abnormalities and vesiculation of the inner membranes (Fig 2C).

The structural abnormalities of the optic nerve axons were not restricted to the Opa $1^{+/-}$mice. Opa $1^{+/+}$mice also underwent age-related degeneration of the axons. With increasing age, there was a significant increase in the percentage of axons with watery degeneration (Fig. $4 \mathrm{~A} ; P=0.008$ ) and myelin clumping (Fig. 4C; $P=0.013$ ). At 24 months, two $O p a 1^{+/+}$mice and one Opa $1^{+/-}$mouse had extensive demyelination of the optic nerve. Opa $1^{+/+}$and $O p a 1^{+/-}$mice experienced significant reductions in the number of axons per area of optic nerve with increasing age (Fig. $4 \mathrm{E} ; P=0.009$ and $P=0.002$, respectively) indicating age-related axonal loss.

\section{Retina}

Almost all the mitochondria in the RGCs had clear morphology, though there was an occasional mutant mitochondrion in the 24-month-old $\mathrm{Opa1} 1^{+/-}$mice, as seen in the optic nerve axons.

At 6 months, there was no difference in the number of autophagosomes in the RGCs and surrounding axons between $O p a 1^{+/-}$and $O p a 1^{+/+}$. At 24 months, however, there were significantly more autophagosomes in the $O p a 1^{+/-}$mice than in the $O p a 1^{+/+}$mice $\left(4.25 \pm 1.38\right.$ vs. $2.57 \pm 0.17 \mu \mathrm{m}^{-2} ; P=$ 0.016 ; Fig. 3B). The number of autophagosomes in the 24month-old $\mathrm{Opal}^{+/+}$mice was significantly lower than in the 6 -month-old control mice $(P=0.001)$. There were no statistically significant age-related differences in the $O p a 1^{+/-}$mice.

\section{Discussion}

In this study, we describe structural abnormalities in the optic nerve and the RGC layer of an Opa1 $1^{+/-}$mouse model of ADOA. We previously published data showing abnormalities in the myelination of the optic nerve in 9- and 18-month-old Opa $1^{+/-}$mice, ${ }^{12}$ and our data here confirm that this is the case up to 24 months of age.

Two types of axonal degeneration have been described in the literature. ${ }^{13,14}$ One is dark degeneration, in which the axons have a dense axoplasm possibly because of neurofilament aggregation, ${ }^{15}$ and the other is watery degeneration, in which the axoplasm either is empty or is filled with an amorphous granular material. Both types of degeneration were seen in the Opa1 $1^{+/-}$and $O p a 1^{+/+}$mice, though watery degeneration was more prominent. The age-related changes seen in the control mice made it difficult to remove structural abnormalities caused by the OPA1 mutation and those caused by the aging process. However, we hypothesized that from the time abnormalities appear in the OPa $1^{+/-}$mice, the OPA1 mutation results in premature aging of the optic nerve, and that control mice might eventually catch-up with $\mathrm{Opa} 1^{+/-}$mice in axonal degeneration.

The age-related changes seen in the optic nerves of our control mice are similar to those reported in the optic nerves of rhesus monkeys, ${ }^{16}$ in which the most prominent changes were axonal degeneration (both dark and watery) and reduced numbers of nerve fibers. However, the extensive demyelination found in two of our controls and in one $O p a 1^{+/-}$mouse was unexpected and not paralleled by similar levels of demyelination in the optic nerves of humans ${ }^{17}$ or monkeys. ${ }^{16}$

The presence of two populations of mitochondria (clear and opaque) in optic nerve axons has been observed recently by our group in an Opa3 mutant mouse model of Costeff syndrome in humans. ${ }^{18}$ However, given that both types are present in $\mathrm{Opa}^{+/-}$and $\mathrm{Opa1} 1^{+/+}$mice (though the opaque type is more prevalent in $O p a 1^{+/-}$mice), they may simply represent an increase in the density of cristae to fulfill the energy requirements of the axon.

Perhaps of more relevance was the observation that some mitochondria in the older Opa1 $1^{+/-}$mice had an abnormal cristae structure similar to that of mutant mitochondria described in a mouse model of Charcot-Marie-Tooth type $2 \mathrm{~A} .{ }^{19}$ This neurodegenerative disease is caused by mutations in the mitochondrial fusion gene $M f n 2 .{ }^{20} \mathrm{Mfn} 2$ is one of the three GTPases involved in mitochondrial fusion. ${ }^{10}$ The others are Mfn1 and OPA1. ' Thus, it is highly feasible that mutations in OPA1 could cause morphologic changes to the mitochondria similar to those seen with mutations in Mfn2.

We also looked at the structure of the RGCs, and, though a few mitochondria had abnormal cristae organization, the most prominent feature of the RGCs and the surrounding axons was the presence of several double membrane-bound structures that were identified as autophagosomes. ${ }^{21}$ Although biomarkers have been used to identify autophagosomes, this method is compromised because of a lack of differential gene expression; transmission electron microscopy remains the most accurate way to evaluate autophagy in tissue. ${ }^{22}$ Even so, electron microscopy is not without its limitations, and it is possible to overestimate autophagy by misidentifying other vacuoles as autophagic. In our study, we counted only structures that were bound by a double membrane or were obviously fusing with a lysosome. We did not try to count late-stage autophagic vacuoles because these cannot be distinguished from heterophagic vacuoles. Given that large numbers of nonspecific vacuoles were present in the cells, we feel we were more likely to have underestimated rather than overestimated the level of autophagy occurring in these cells.

Autophagy is a cellular pathway for the clearance of proteins and organelles; therefore, some degree of autophagy is necessary to maintain normal cellular homeostasis. ${ }^{23}$ Different tissues require different levels of autophagy, but it is particularly important in quiescent cells such as neurons. ${ }^{24}$ Thus, it was not surprising that autophagosomes were observed in the RGCs of our mice. In addition, the reduction in the number of autophagosomes in the older control mice is consistent with the published data on autophagy decline with age. ${ }^{25,26}$

What is even more interesting is that there were more Opa $1^{+/-}$mice than littermate controls. In addition, there was no age-related decline in autophagy in the $O p a 1^{+/-}$mice. Increased numbers of autophagosomes are a feature in a number of neurologic diseases, but the question is whether this is a result of the increased formation of autophagosomes or of the decreased lysis and breakdown of the autophagosomes. ${ }^{27}$ The large amounts of nonspecific vacuoles and cytoplasmic debris that were also present suggest that the formation of autophagosomes was increased in this study.

Why then would a mutation in OPA1 cause increased autophagy? Downregulation of OPA1 results in a loss of the mito- 
chondrial membrane potential and, thus, the formation of depolarized mitochondria, which are dysfunctional and must be "rescued" or eliminated. It has been suggested that mitochondrial fusion is a mechanism by which depolarized mitochondria are recovered, ${ }^{28}$ whereas autophagy has been shown to target depolarized mitochondria for digestion and elimination. ${ }^{29,30}$ If fusion and autophagy compete with each other for depolarized mitochondria in our Opa $1^{+/-}$mouse model, in which mitochondrial fusion is impaired, autophagy is more likely to be the winner. This hypothesis is supported by a recent study in which overexpression of OPA1 decreased mitochondrial autophagy. ${ }^{31}$

It could be argued that this increased autophagy would maintain cellular homeostasis and have no detrimental effect on the cell. However, because RGC loss is the primary pathologic characteristic of ADOA, it is possible that the autophagic process becomes overloaded, resulting in an accumulation of defective mitochondria, the release of cytochrome $c$, and ultimately the apoptosis of RGCs.

In conclusion, we have shown that mutations in OPA1 cause pathologic changes to optic nerve axons that are similar to, but occur earlier than, age-related degeneration. In addition, we suggest that increased autophagy could be one mechanism contributing to RGC loss and the subsequent optic atrophy seen in ADOA.

\section{Acknowledgments}

The authors thank Tracey Davey and Vivian Thompson (EM Research Services, Newcastle University, UK) for technical assistance.

\section{References}

1. Kjer P. Infantile optic atrophy with dominant mode of inheritance: a clinical and genetic study of 19 Danish families. Acta Opbthalmol. 1959;164(suppl 54):1-147.

2. Kline LB, Glaser JS. Dominant optic atrophy: the clinical profile. Arch Opbthalmol. 1979;97:1680-1686.

3. Votruba M, Moore AT, Bhattacharya SS. Clinical features, molecular genetics, and pathophysiology of dominant optic atrophy. J Med Genet. 1998;35:793-800.

4. Johnston PB, Gaster RN, Smith VC, Tripathi RC. A clinicopathologic study of autosomal dominant optic atrophy. Am J Opbthalmol. 1979;88:868-875.

5. Kjer P, Jensen OA, Klinken L. Histopathology of eye, optic nerve and brain in a case of dominant optic atrophy. Acta Opbthalmol (Copenb). 1983;61:300-312.

6. Alexander C, Votruba M, Pesch UE, et al. OPA1, encoding a dynamin-related GTPase, is mutated in autosomal dominant optic atrophy linked to chromosome 3q28. Nat Genet. 2000;26:211215.

7. Delettre C, Lenaers G, Griffoin JM, et al. Nuclear gene OPA1, encoding a mitochondrial dynamin-related protein, is mutated in dominant optic atrophy. Nat Genet. 2000;26:207-210.

8. Olichon A, Emorine LJ, Descoins E, et al. The human dynaminrelated protein OPA1 is anchored to the mitochondrial inner membrane facing the inter-membrane space. FEBS Lett. 2002;523:171176.

9. Cipolat S, Martins de Brito O, Dal Zilio B, Scorrano L. OPA1 requires mitofusin 1 to promote mitochondrial fusion. Proc Natl Acad Sci U S A. 2004;101:15927-15932.

10. Chen H, Detmer SA, Ewald AJ, Griffin EE, Fraser SE, Chan DC. Mitofusins Mfn1 and Mfn2 coordinately regulate mitochondrial fusion and are essential for embryonic development. J Cell Biol. 2003;160:189-200.
11. Olichon A, Baricault L, Gas N, et al. Loss of OPA1 perturbates the mitochondrial inner membrane structure and integrity, leading to cytochrome $c$ release and apoptosis. J Biol Chem. 2003;278:77437746.

12. Davies VJ, Hollins AJ, Piechota MJ, et al. Opa1 deficiency in a mouse model of autosomal dominant optic atrophy impairs mitochondrial morphology, optic nerve structure and visual function. Hum Mol Genet. 2007;16:1307-1318.

13. Cook RD, Ghetti B, Wisniewski HM. The pattern of Wallerian degeneration in the optic nerve of newborn kittens: an ultrastructural study. Brain Res. 1974;75:261-275.

14. Narciso MS, Hokoc JN, Martinez AM. Watery and dark axons in Wallerian degeneration of the opossums optic nerve: different patterns of cytoskeletal breakdown? Anais Acad Brasileira Ciencias. 2001;73:231-243.

15. Marques SA, Taffarel M, Blanco Martinez AM. Participation of neurofilament proteins in axonal dark degeneration of rats optic nerves. Brain Res. 2003;969:1-13.

16. Sandell JH, Peters A. Effects of age on nerve fibers in the rhesus monkey optic nerve. J Comp Neurol. 2001;429:541-553.

17. Giarelli L, Grandi G, Delendi M, Falconieri G. The pathology of optic nerve aging. Metab Pediatr Syst Opbthalmol. 1989;12:6163.

18. Davies VJ, Powell KA, White KE, et al. A missense mutation in the murine Opa3 gene models human Costeff syndrome. Brain. 2008; 131:368-380.

19. Chen H, McCaffery JM, Chan DC. Mitochondrial fusion protects against neurodegeneration in the cerebellum. Cell. 2007;130:548562.

20. Zuchner S, Mersiyanova IV, Muglia M, et al. Mutations in the mitochondrial GTPase mitofusin 2 cause Charcot-Marie-Tooth neuropathy type 2A. Nat Genet. 2004;36:449-451.

21. Dunn WA Jr. Studies on the mechanisms of autophagy: formation of the autophagic vacuole. J Cell Biol. 1990;110:1923-1933.

22. Martinet W, De Meyer GR, Andries L, Herman AG, Kockx MM. Detection of autophagy in tissue by standard immunohistochemistry: possibilities and limitations. Autophagy. 2006;2:55-57.

23. Mizushima N, Levine B, Cuervo AM, Klionsky DJ. Autophagy fights disease through cellular self-digestion. Nature. 2008;451:10691075.

24. Hara T, Nakamura K, Matsui M, et al. Suppression of basal autophagy in neural cells causes neurodegenerative disease in mice. Nature. 2006;441:885-889.

25. Del Roso A, Vittorini S, Cavallini G, et al. Ageing-related changes in the in vivo function of rat liver macroautophagy and proteolysis. Exp Gerontol. 2003;38:519-527.

26. Terman A. The effect of age on formation and elimination of autophagic vacuoles in mouse hepatocytes. Gerontology. 1995; 41(suppl 2):319-326.

27. Rubinsztein DC, DiFiglia M, Heintz N, et al. Autophagy and its possible roles in nervous system diseases, damage and repair. Autophagy. 2005;1:11-22.

28. Chan DC. Mitochondria: dynamic organelles in disease, aging, and development. Cell. 2006;125:1241-1252.

29. Elmore SP, Qian T, Grissom SF, Lemasters JJ. The mitochondrial permeability transition initiates autophagy in rat hepatocytes. FASEB J. 2001;15:2286-2287.

30. Priault M, Salin B, Schaeffer J, Vallette FM, di Rago JP, Martinou JC. Impairing the bioenergetic status and the biogenesis of mitochondria triggers mitophagy in yeast. Cell Death Differ. 2005;12:16131621.

31. Twig G, Elorza A, Molina AJ, et al. Fission and selective fusion govern mitochondrial segregation and elimination by autophagy. EMBO J. 2008;27:433-446. 